\title{
ESCUCHAR PARA SOSTENER LA FUNCIÓN DE EDUCAR APORTES PARA PENSAR LA FUNCIÓN DE EQUIPOS DIRECTIVOS Y PROFESORES DE ORIENTACIÓN Y TUTORÍA EN EDUCACIÓN SECUNDARIA
}

\author{
Irma Kundt* \\ Facultad Latinoamericana en Ciencias Sociales, Argentina \\ irmakundt@yahoo.com.ar
}

Recibido: 16/08/2019 Aceptado: 23/10/2019

\section{Resumen}

El presente escrito tiene como objetivo comunicar las reflexiones que suscita el recorte de una experiencia escolar signada por los desencuentros con el otro en la tarea de hacer equipo para sostener la función de educar.

Mediante una narrativa etnográfica se ha podido arribar a la construcción de un caso que permite alumbrar el problema de la escucha y el contenido de una demanda haciendo uso de las contribuciones de Lacan en relación con los discursos y su rotación. Algunos de los interrogantes que se suscitaron fueron: ¿Cómo hacer equipo y sostener la función educadora entre adultos que asumen diferentes roles cuando hay creciente malestar entre ellos?

El recorrido propuesto se inicia con el relato de alguna/s escena/s de la práctica, a partir de los cuales se describe el punto de partida de la construcción del caso. Desde aquí, se ofrece una lectura de $\mathrm{la} / \mathrm{s}$ escena/s desde tres dimensiones de análisis: subjetiva, institucional y socio-histórico/cultural. Finalmente, se propone arribar a un dispositivo de trabajo producto de las conclusiones provisoras alcanzadas alrededor del núcleo problemático que interroga o desafía la práctica del profesional en Ciencias de la educación en la dirección de una escuela.

Palabras Clave: Encuentro - Discurso - Función - Escucha.

\begin{abstract}
This paper of writing leads to communicate thoughts about a scholar experience in which disagreement among educators, who have to fulfil the function of educacting, has an impact on the educational matter.

Through an etnographic narrative I could analize a case study that allows to see the matter of listening and the content of a request considering the contributions of Lacan related to those discourses.

*Especialista en Ciencias Sociales con mención en Psicoanálisis y prácticas socio educativas - FLACSO-2019 Especialista en Ciencias Sociales con mención en Constructivismo y Educación- FLACSO -UAM 2007. Diplomada en Gestión de las Instituciones educativas. FLACSO. Profesora y Licenciada en Ciencias de la Educación. UNPSJB. Profesora adjunta de la cátedra Psicología Evolutiva I. Universidad Nacional de la Patagonia San Juan Bosco. Profesora adjunta de la cátedra Historia Social de la educación. Universidad Nacional de la Patagonia San Juan Bosco. Actualmente Directora Interina de Escuela Secundaria Provincial № 732. Autora de Libros: (2012); Habitar y hacer escuela; Editorial Vela Al Viento. Buenos Aires. (2015); Pensar la escuela desde abajo; Editorial Mis Escritos. Buenos Aires. Supervisora Técnica de educación secundaria: 2014-2016.
\end{abstract}


REVISTA DE LA ESCUELA DE CIENCIAS DE LA EDUCACIÓN, AÑO 16, NRO. 15, VOL. 1, ENERO A JUNIO DE 2020. PÁGINAS 73 - 83. ISSN 2362-3349 (EN LÍNEA). ESCUCHAR PARA SOSTENER LA FUNCIÓN DE EDUCAR APORTES PARA PENSAR LA FUNCIÓN DE EQUIPOS DIRECTIVOS Y PROFESORES DE ORIENTACIÓN Y TUTORÍA SECUNDARIA. IRMA KUNDT.

Some questions have emerged such as: How to do team and hold the educational matter among adults who have different roles in a context of Deep confort?

This research starts describing some situations of the fieldwork. Thus, readers can analize three dimensions: subjective, institutional, socio-historical and cultural. Finally, the aim of this study is to devise a method for woking arised from drawing conclusions related to the heart of the matter that challenges the fieldwork in Educational discipline, specifically in the function of conducting schools.

Keywords: Meeting - Discourse - Function - Listening.

\section{Introducción}

En este trabajo abordo un malestar entre adultos que integran equipos docentes, suscitado en relación con la escucha, el rol y las funciones. Comunico los hallazgos de una clínica psicoanalítica en torno a la construcción de un caso, empleo una narrativa etnográfica y autobiográfica.

Impele para la formación de docentes en cargos directivos en general, y de profesionales en Ciencias de la educación en particular, cuando asumen estas responsabilidades, pensar las dificultades para sostener la función educadora en el escenario de la educación pública, la práctica de filiación y lazo con la cultura sostenida entre varios/as, las estrategias de recibimiento y hospitalidad hacia quienes integran los equipos y sus efectos de alojamiento.

\section{Escena y escenario de un desencuentro}

Trabajo con el malestar de alguien que en esta escritura llamaré Adriana. Su desempeño es en el cargo de Profesora de Orientación y Tutoría (POT).

Adriana no acuerda con el encuadre de trabajo y con parte de las tareas asignadas en una institución en la que me desempeño como directora de escuela. En su último año de trabajo no siempre asiste a las reuniones institucionales. Suele presentarse como porta voz de lo que piensan sus pares "esto que te digo lo pensamos todas"; "no estamos de acuerdo con formar parejas pedagógicas", y ante los cambios de espacios curriculares extiende la idea: "no estamos de acuerdo con formar parejas pedagógicas en las disciplinas para las que no tenemos formación específica"; "todas vamos a renunciar". Manifiesta no acordar con un dispositivo que desde el año 2013 implementamos en el establecimiento: Parejas pedagógicas (PP) y cuya ideación le precede a su ingreso. Su cargo se gestiona ante el Ministerio de Educación precisamente con este proyecto.

Nombro el malestar condensando en su formulación las diferentes expresiones de Adriana: "el problema es que los directivos no escuchan". Durante este proceso emerge también el malestar que nos genera su malestar. Sentimiento recíproco, reiterado.

En el año 2017 ponemos a trabajar el proyecto en cuestión en dos reuniones institucionales para su reescritura o abandono. Decimos: "este es el momento de decidir continuar o abandonar el proyecto y presentar otras ideas". "Si no le damos continuidad ¿qué hacemos?". "¿Cómo seguimos, qué implementamos?".

No escriben modificaciones que las eximan de esta obligación: "no vamos a presentar nada, nos cansamos de presentar proyectos pero no escuchan". Y nunca recibimos esos - otros- proyectos.

Un indicio del mal-estar ha sido el constante pedido de cambio de espacio curricular.

Cuando asignamos integrar pareja pedagógica en Matemáticas esgrime entre los argumentos el siguiente: "cuando fuimos a la escuela (habla en tiempo pasado haciendo alusión a su propia biografía escolar) siempre tuvimos dificultades en esta disciplina y por lo mismo escogimos carreras universitarias que no tenían matemáticas en su formación. No estudiamos matemáticas." El resto del equipo de POT asiente y apoya con comentarios autorreferenciales similares.

Hasta ahí el equipo directivo escucha en relación con el cambio de espacio curricular, efectivizando el cambio y conversando incluso dónde preferían estar o dando lugar al pedido puntual de alguna disciplina; sin dar lugar al pedido "no hacer pareja pedagógica". Luego, cuando el pedido lo formulan un año tras otro, con cada espacio curricular diferente, incluso durante el transcurso de la experiencia, algo se quiebra. Aparece una y otra vez lo mismo si el espacio es Ciencias Sociales, Tecnología, Lengua, Formación Ciudadana, Inglés, Lenguajes artísticos. Sin visualizar que dada la falta de proximidad con su formación de base, en reiteradas veces la dirección da lugar al pedido de Adriana y también de las restantes POT, con lo que inician por años un itinerario por diferentes espacios y con distintos/as docentes sin lograr que en alguno accedan a la tarea abandonando la queja.

Los/as profesores/as que a su vez debían sentir el acompañamiento a la enseñanza no lo experimentan y de algún modo empiezan a manifestar que no estén y a verbalizar lo mismo que de ellas escuchan: "¿Cómo pueden ocuparse del aula acelerada " $x$ " si no saben?" (Como si no supieran nada de nada) esto cuando tenían que diseñar e implementar algún dispositivo alternativo para reingresar estudiantes e idear situaciones de enseñanza novedosas e integradas.

Otro planteo de Adriana estaba dirigido a no integrar PP porque les restaba tiempo para ocuparse de emergentes importantes y problemas específicos que atraviesan los/as chicos/as, y a causa de ello decía que 
REVISTA DE LA ESCUELA DE CIENCIAS DE LA EDUCACIÓN, AÑO 16, NRO. 15, VOL. 1, ENERO A JUNIO DE 2020. PÁGINAS 73 - 83. ISSN $2362-3349$ (EN LÍNEA). ESCUCHAR PARA SOSTENER LA FUNCIÓN DE EDUCAR APORTES PARA PENSAR LA FUNCIÓN DE EQUIPOS DIRECTIVOS Y PROFESORES DE ORIENTACIÓN Y TUTORÍA SECUNDARIA. IRMA KUNDT.

los desatendían; refería a situaciones de abusos entre otras cuestiones. No me ocuparé aquí de extender o explicar estas ideas; las explicito para dar cuenta de diferentes razonamientos para no integrar PP.

\section{POT en educación secundaria}

El cargo POT fue creado para atender a las necesidades de los estudiantes del Tercer ciclo de la educación secundaria Básica. En muchas escuelas de la Provincia de Chubut estuvo primarizado hasta el año 2010. En correspondencia con ese nivel se empleó la sigla MOT (maestro) y la organización del trabajo propio de estas funciones siguió la lógica de funcionamiento de las escuelas primarias: cargo de 20 horas semanales a cumplir distribuidas proporcionalmente en los cinco días de la semana. Esto se ha diferenciado de la organización tradicional de la planta orgánica en educación secundaria para el resto de los profesores: designaciones por horas cátedras y frente a alumnos.

A partir del año 2008, con la implementación de la Ley Nacional de Educación 26.206 y Ley Provincial VIII № 91 las escuelas han ido incorporando estos cargos haciéndolos extensivos a todos los años de la educación secundaria.

A los docentes que se desempeñan en cargos de POT, siguiendo el mandato de la inclusión, se les formula entre otros el encargo de acompañar y sostener las trayectorias escolares de alumnos/as, su continuidad y egreso, lo que adquiere cierta peculiaridad para el caso, en tanto tienen la posibilidad de una mayor presencia en la escuela. Se ocupan de detectar situaciones que afectan y vulneran derechos, e intervenir, en el marco de sus obligaciones para su restitución. Analizan trayectorias escolares, integran mesas de gestión y comisiones de acreditación, idean y ejecutan proyectos integrados, participan del diseño de proyectos pedagógicos individuales (solo en caso de necesidad y de estudiantes con diagnóstico), gestionan escuela hospitalaria para alguien en situación de enfermedad, crean e implementan dispositivos alternativos para diferentes recorridos cuando la escolaridad se ve interrumpida y/o en riesgo.

\section{РОT en la singularidad de la escuela $A^{1}$}

En la Escuela A que gestiono como directora sostenemos la siguiente organización para los POT: del total de 20 horas reloj ${ }^{2}$ cada agente debe emplear un mínimo de ocho horas cátedra frente a alumnos/as, integrando PP con otros/as docentes; esto debido a que su designación es por cargos y en este caso no tienen horas de clases en la estructura curricular, paradójicamente, lo que dificulta la generación de un vínculo con los estudiantes. La consigna inicial para conformar las PP era "en aquellos espacios donde se presente la mayor cantidad de estudiantes en riesgo".

En el año 2012 el Ministerio de Educación de la Provincia del Chubut sentó las bases para el trabajo colaborativo interinstitucional. La Escuela A integró una red conformada también por otros establecimientos educativos secundarios públicos (B, C, D). De esta experiencia de pensamiento colectivo en torno a problemas comunes surgieron los proyectos Pareja Pedagógica y Programa para alumnos repitentes con sobre edad, a partir de los cuales en cada institución se debía reorganizar el trabajo del personal, entre ellos el de los equipos de POT, fortaleciendo con estos profesionales los espacios en los que los/as estudiantes presentaban mayores dificultades. Las Escuelas que no contaban con este personal en proporción a su matrícula gestionaron el ingreso de profesionales en cargos de POT, tal es el caso de la Escuela A. En este sentido la PP y el programa para estudiantes en riesgo enmarcaron e imprimieron su impronta al trabajo de los POT en estas escuelas en particular. Es importante destacar esto pues sin la ideación de estos proyectos no se habrían gestionado los cargos, por lo tanto es llamativo para el equipo directivo que quienes ingresan manifiesten renuencias a las tareas que se solicitan: "Esto no nos corresponde hacer (referido a estar en el aula y enseñar) porque no nos pagan la hora cátedra" se suele con frecuencia escuchar, y verbalizaba también Adriana.

En los últimos años, 2017 y 2018, y en la Escuela A, estos trayectos alternativos adquieren diferentes formatos y nominaciones: Aula acelerada ${ }^{3}, \mathrm{ClIC}^{4}$, Dispositivo de colaboración cruzada ${ }^{5}$. La hipótesis común

\footnotetext{
${ }^{1}$ A los efectos de esta presentación emplearé letras del alfabeto español en mayúsculas para guardar reserva de la identidad institucional.

${ }^{2}$ Consigno hora reloj porque la duración predominante en educación secundaria no es de sesenta minutos sino de cuarenta (hora cátedra) y destinadas a la enseñanza de contenidos de la estructura curricular organizada por disciplinas, y en un porcentaje inferior por áreas. Solo en la designación por cargos, como preceptor/a, PEP y POT cada hora de trabajo dura sesenta minutos pero no las poseen frente a estudiantes en el actual diseño curricular, por lo mismo es una impronta singular en la escuela A prever esta presencia en aulas mediante la organización de PP.

${ }^{3}$ Se ideó e implementó en 2017. Adoptó una modalidad integrada e intensiva al igual que el CIIC pero desarmando la tradicional organización de horarios que programa la cursada de varios espacios en cada jornada y semana escolar. A diferencia de ello por una o dos semanas los alumnos tenían una jornada completa con un mismo módulo. Se dictaron cuatro en forma consecutiva y no simultánea.

${ }^{4}$ CIIC: Cursada integrada intensiva cuatrimestral. La sigla nomina al trayecto diseñado para facilitar el aprendizaje, evaluación y acreditación de estudiantes que es necesario reingresar en un curso consecutivo superior para no quedar rezagados en relación a sus grupos. Este dispositivo es de ideación reciente, una invención del equipo de POT, PEP, algunos profesores y directivos de la Escuela A. Al momento de esta escritura todos los integrantes del equipo POT turno mañana son nuevos en el establecimiento, han ingresado a inicios de ciclo lectivo 2018.
} 
REVISTA DE LA ESCUELA DE CIENCIAS DE LA EDUCACIÓN, AÑO 16, NRO. 15, VOL. 1, ENERO A JUNIO DE 2020. PÁGINAS 73 - 83. ISSN 2362-3349 (EN LÍNEA). ESCUCHAR PARA SOSTENER LA FUNCIÓN DE EDUCAR APORTES PARA PENSAR LA FUNCIÓN DE EQUIPOS DIRECTIVOS Y PROFESORES DE ORIENTACIÓN Y TUTORÍA SECUNDARIA. IRMA KUNDT.

es que reiteradas repitencias pone a los/as estudiantes en mayor riesgo de abandonar la escolaridad cuando quedan rezagados y el grupo de pares que opera como núcleo de referencia avanza sin ellos/as.

La persistencia del equipo directivo en sostener el encuadre radica en lo necesario que es para los/as estudiantes la filiación desde el vínculo educativo que debe ser forjado y sostenido (Hebe Tizzio; Violeta Núñez: 2003) lo que en estos tiempos sólo es posible en el lazo y en la práctica entre varios para: fortalecer la enseñanza haciendo equipo, no interrumpir las rutinas escolares, cubrir las horas de clase cuando se suscita la ausencia de un docente, evitar horas libres y propiciar su empleo pluralizando la oferta de experiencias subjetivantes. Estos lineamientos y fundamentos se ponen a trabajar y socializan entre los/as integrantes del equipo en diferentes reuniones específicas.

\section{¿Cómo hacer lugar a aquello que por inesperado no se lo esperaba en ningún lugar?}

(Balbi y Serravalle; 2014, Clase 2, Ap. II).

Adriana renunció. En su ausencia, aunque sea posible rotar en el discurso ya no es posible su ingreso en otro lazo, en otro discurso, en otros encadenamientos significantes (Zelmanovich, 2014, Clase 4, Ap: I), no en esta escuela, no en este equipo.

Reconociendo el papel simbólico estructurante del lenguaje que precede al sujeto, la rotación en el discurso puede ser pensada como un dispositivo de intervención que ayuda a profesionales de la educación a sostener la función alternando las posiciones propias cambiando de semblantes, es decir alternar los lugares que se ocupan dentro de una estructura discursiva, generando con ello los lugares a ocupar por otros/as. Podría ser también que en la rotación se propicie el ingreso de otros/as profesionales en el lazo (una práctica entre varios), para favorecer al semejante en cuestión que se ubique en un lugar también diferente en relación al que venía ocupando y que este nuevo lugar no resulte mortificante y no lo fije en la estructura, alienado/a o condenado/a a la repetición.

Los cuatro discursos escriben cuatro modos de producir lazos sociales, cuatro maneras de intentar encontrarse con el otro, y de producir puntos de fracaso, de imposibilidad de regularlo todo, lo cual habilita el pasaje de un discurso al otro, allí donde se encuentra cada discurso con su propio límite. Su valor de herramienta se ancla en la posibilidad de leer y advertir una posición desde la cual un profesional puede ubicar a un sujeto, lo cual abre un cálculo posible para un trabajo sobre dicha posición cuando la relación educativa se ve interferida y declina la función, lo cual facilita el ingreso del sujeto en un circuito de segregación (...) Cuatro discursos: del amo, universitario, Discurso del sujeto Histérico, Discurso del analista. (Zelmanovich, 2013 [2010]), Flacso, Clase N 6, Ap.: III).

La formalización de los discursos de Lacan es recuperada por Perla Zelmanovich (2010) en tanto permite ubicar cómo el goce encuentra su lugar particular en los lazos sociales según de qué discurso se trate. Define a los discursos como las formas por medio de las cuales se construyen y sostienen los lazos sociales y toda articulación entre el sujeto y el lenguaje.

Pienso esta idea de la rotación como sostén del lazo social; lo contrario sería la fijeza en las posiciones. Algo de ello pudo operar en la renuncia de Adriana si la pensamos en términos de producción, definido como el lugar ocupado por aquello que es efecto de lo que el Otro produce en un tipo de lazo particular (Zelmanovich; 2013 [2010]), Flacso, Clase $N^{\circ}$ 6, Ap.: III). Las expectativas iniciales en relación al encuadre de trabajo y las representaciones negativas que fuimos construyendo, a partir de sus quejas, no favorecieron que pase de un régimen de representaciones a otro y que pueda ingresar en otra cadena significante como un interlocutor diferente del que era. (Zelmanovich; 2013 [2010]), Flacso, Clase N 6, Ap.: II)

\section{El contenido en la literalidad de los dichos. De lo manifiesto a lo latente}

"Me dirijo a Ud. a efectos de informarle la RENUNCIA al cargo de POT del turno mañana, ciclo básico. Los motivos se basan en los desacuerdos y malestares suscitados con los lineamientos vigentes en las funciones del POT"6

\footnotetext{
${ }^{5}$ Supone la posibilidad de trabajo colaborativo entre equipos que no coinciden en un mismo turno pero que pueden organizar a los alumnos propios, los del turno mañana por ejemplo, para asistir a la biblioteca en turno tarde y viceversa. Eso implica una extensión del tiempo escolar para hacer tareas, leer libros, completar carpetas. En la escuela A, el ciclo básico de tres años funciona en turno mañana y el ciclo orientado (4to, 5to, 6to) en turno tarde. La propuesta inicial era colaboración cruzada de bibliotecarios/as y POT de 1 er año con POT de 4to, POT de 2do con POT de 5to y de 3ro con 6 to. En 2019 se abandonó la experiencia y cada POT flexibilizó su propio horario para cumplir parte del mismo a contra turno de la escolaridad de los/as estudiantes y así poder brindar las ayudas. Si bien resulta bueno en relación al grupo de estudiantes asignado, también podría ser un signo de la dificultad para hacer equipos.

${ }^{6}$ El destacado en mayúsculas pertenece a Adriana en su nota de renuncia transcripta literalmente.
} 
REVISTA DE LA ESCUELA DE CIENCIAS DE LA EDUCACIÓN, AÑO 16, NRO. 15, VOL. 1, ENERO A JUNIO DE 2020. PÁGINAS 73 - 83. ISSN $2362-3349$ (EN LÍNEA). ESCUCHAR PARA SOSTENER LA FUNCIÓN DE EDUCAR APORTES PARA PENSAR LA FUNCIÓN DE EQUIPOS DIRECTIVOS Y PROFESORES DE ORIENTACIÓN Y TUTORÍA SECUNDARIA. IRMA KUNDT.

Si bien la nota expresa el motivo, por qué renuncia, no obstante inicialmente le atribuí un significado taponando cualquier otra interpretación: "por no conseguir que desistamos del encuadre que no acepta" (me dije).

Tiempo después lo considero distinto. Si en la renuncia veo un indicio del desencuentro, es allí donde me percibo implicada. En el vínculo que la anticipa y no en la renuncia está el enigma, como una crónica de muerte anunciada, parafraseando a García Márquez.

Los motivos del desencuentro no están claros pese a las racionalizaciones (conscientes) esgrimidas; de una parte y de otra operaron como mecanismo de defensa. El trabajo de desanudamiento es ubicar lo inconsciente como lo que no se sabe... un saber que no se sabe es un saber a producir, tal como lo precisa Zelmanovich. Y, no obstante, debo recordar el punto de imposibilidad de todo análisis: "algo siempre queda en penumbras, en las sombras aún del propio sujeto. Freud lo llamó inconsciente” (Zelmanovich; 2014, Clase 13, Ap: I).

\section{Se espera lo inesperado puesto que el encuentro no será sin contingencias}

(Balbi y Serravalle; 2014, Clase 2, Ap. II).

Balbi y Serravalle retoman la obra de Freud, el Malestar en la cultura, y nos explican que por estructura los humanos nos enfrentamos a distintos embates; entre ellos los que vienen del mundo o la naturaleza, los que se suscitan en el encuentro con los semejantes, y los embates pulsionales que vienen del cuerpo (2014, Clase 2, Ap. I)

Consultando el diccionario español encontramos que la palabra embate remite a "Golpe fuerte contra algo". "Acometida o ataque impetuoso"? . Entre los sinónimos: irrupción, asalto, carga.

Este embate se trata del encuentro que no se produjo. Al trabajarlo siento la tensión que me provocó como directora de escuela, sostener un marco organizativo pensado para dar sentido a lo institucional instituido para todos y sostener en la tarea a alguien, quien sea, Adriana en este caso, cuando era insostenible sin su anuencia, sin su deseo.

Sigmund Freud explica el desencuentro ineludible entre la necesidad de la satisfacción pulsional y la respuesta encontrada. Pero si encontramos en los hombres la presencia de un malestar estructural, como "un vacío imposible de suturar", si este desencuentro no sólo genera el malestar sino también la cultura, reconforta saber y sentir que también allí radica su potencia: "porque es imposible la satisfacción total de la pulsión, hay búsqueda, hay producción, hay cultura, hay deseo" (Zelmanovich; 2014, Clase 13, Ap. I).

Hay proyectos marco y criterios de evaluación que regulan el ingreso de un/ POT a la escuela $A$. Quizás, en adelante, cuando pensamos la función de este cargo y delineamos lo que esperamos en su ejercicio nos será oportuno tener presente que:

(...) este encuentro de expectativas no puede ser pensado sin las vicisitudes que en todo encuentro acaecen. No hay correspondencia entre lo que se va a buscar y lo que se encuentra, entre lo que se espera y recibe. Cualquier encuentro conlleva un desencuentro, por estructura, habrá también lo que rebase lo reglado, lo esperado, institucionalizado (...) Habrá que estar dispuestos a considerar la paradoja, el inevitable encuentro con lo contingente que se presenta como inesperado (Balbi y Serravalle; 2014, Clase 2, Ap. II).

\section{En búsqueda de un significado}

Pensé que a Adriana la mortificó sentir "no saber", no saber algo, ¿saber nada? ¿Matemáticas, Historia, Ciencias Sociales, Tecnología, Lengua, Construcción y ciudadanía, Inglés, Lenguajes artísticos?

No es que yo pensara que alguien debe tener dominio en diferentes campos de conocimiento. El equipo directivo asume que ese conocimiento específico puede no saberse, lo que no consiente es un no saber- poder hacer equipo en torno a un contenido: el contenido " $X$ " en sentido amplio como algo a enseñar, algo que media en el vínculo educativo entre docentes y alumnos y hace a la especificidad de una escuela, porque es precisamente de la función docente que esto se trata ¿No saber- no poder, con unos u otros, en alguna PP?

Me incomodó su posición. Sentía que declinando en su función se destituía, se presentaba desvalida ante sus interlocutores. Si construía con su discurso el lugar propio y el de los demás ¿cuál sería mi responsabilidad? Es decir ¿en qué lugar la había ubicado (yo) para obtener este efecto o producción en el lazo?

En un discurso el valor que tiene cada elemento es efecto de la estructura en la que se ubica, no tiene un sentido per se. Es por ello que cuando un Agente habla no sólo ofrece el contenido del mensaje, sino que construye a su interlocutor (...) El interlocutor es así producido en una estructura

\footnotetext{
${ }_{7}^{7}$ Disponible en: https://educalingo.com/es/dic-es/embate. Fecha de consulta: 08/12/18.
} 
REVISTA DE LA ESCUELA DE CIENCIAS DE LA EDUCACIÓN, AÑO 16, NRO. 15, VOL. 1, ENERO A JUNIO DE 2020. PÁGINAS 73 - 83. ISSN 2362-3349 (EN LÍNEA). ESCUCHAR PARA SOSTENER LA FUNCIÓN DE EDUCAR APORTES PARA PENSAR LA FUNCIÓN DE EQUIPOS DIRECTIVOS Y PROFESORES DE ORIENTACIÓN Y TUTORÍA SECUNDARIA. IRMA KUNDT.

determinada, aunque cabe advertir que ello es posible en la medida que por sus propias características y por su lugar institucional se aviene a asumirlo (consciente e inconscientemente) (Zelmanovich; 2013 [2010]), Flacso, Clase №6, Ap.: III).

A partir de los aportes de Graciela Kait (2014), indago en la concepción de sujeto que subyace a mi propia práctica como directora de escuela en relación a Adriana y los POT, concepción que supone un modo de concebir el lazo social; y pienso la renuncia al cargo como una producción que se desencadena de esa relación.

Y por otra parte, en la insistencia de la queja debo pensar que "hay otra cosa en juego" (...). Como explica Zelamovich, se puede suponer que en esa insistencia está el Otro que trae consigo el sujeto, que es también el Otro al que se dirige, (y no va dirigido a mí, tampoco a la directora) pero que no está a la vista ni para el propio sujeto. (Zelmanovich; 2014, Clase 4, Ap: III).

Lo que se presenta como desobediencia, requiere suponer que allí hay un enigma a ser escuchado a partir de la literalidad de los dichos o de las acciones (...). Ese lugar es su decir, que es inconsciente y que trasunta alguna verdad subjetiva con respecto a su posición en relación a ese Otro (Zelmanovich; 2014, Clase 4, Ap: I).

¿Es frente a mi posición de amo antiguo o posición de un saber universitario que siempre esgrime razonamientos y no cambia de semblante ${ }^{8}$ (Zelmanovich; 2014) que Adriana siente no saber- no poder? Vale tener presente que un sujeto en sus dichos presenta un modo de estar en el mundo, su relación con las palabras y las cosas, y el grado de posibilidad de hacerse otro lugar, de posicionarse respecto de lo que se le impone (Balbi y Serravalle; 2014, Clase 2, Ap. I).

\section{Sentirme y estar implicada. Impactos en la función directiva}

La renuencia de Adriana a integrar PP me advierte no estar a la altura de la directora ideal. Advierto lo imposible. Imposible que las atribuciones del cargo directivo operen con la eficacia sobre los sujetos con las que operaban las normas de antaño. O que la palabra, el orden simbólico opere por arte de magia y entonces yo pueda poner a todos a trabajar en armonía, sin incomodidades, sin objeciones.

Rinaldo Voltolini (2014) explica que la realidad institucional necesita, para su concreción y sustento, que los sujetos participen de la danza de los discursos. Nos dice que el pacto civilizatorio subordina la dinámica pulsional al universo de la ley, imprescindible para organizar cualquier institución. "Diluirse en el grupo", parafraseando a este pensador, constituiría una habilidad necesaria para el manejo del discurso del amo, aunque no obstante nunca la expectativa (la mía en este caso) de ser escuchada por todos, podría ser colmada. Por el contrario esta escucha siempre se verá frustrada. Siguiendo sus razonamientos de algún modo es posible pensar que la voz de Adriana constituye en su discordancia la denuncia que "el grupo (el equipo) no es más que un semblante", y por lo mismo es sólo una ilusión que sus integrantes se pondrán a trabajar "al toque" cuando me escuchen. No sucederá. Porta el valor de recordarnos la presencia de lo diferente, lo que es necesario tener presente cada vez a causa de la falaz homogeneidad con la que sin embargo aparenta funcionar la escuela. Asimismo puedo poner en valor su voz, en tanto puede sacarnos de la comodidad de habituarnos a nuestra prisión discursiva y a nuestro goce (Voltolini; 2014, Clase 5, Ap: III).

La PP como uno de los lineamientos de trabajo para los POT son las premisas del discurso del amo. Siguiendo a Voltolini (2014) puedo pensar que Adriana encarnó su reverso. Su desacuerdo constante fue sentido amenazador en la consolidación de un ordenamiento institucional. Al irse, la regla siguió funcionando para los demás y por lo mismo su renuncia trajo al equipo directivo cierto alivio y no obstante el desafío de seguir. Y en lo personal, pensar el contenido de la demanda.

Si el encuadre no cambia, si son la ley del discurso del amo, si representan lo que no se puede abandonar ni trasgredir (el equipo directivo no lo admitirá) entonces es ese Otro que se presenta completo (la directora que brinda unos y otros razonamientos desde un saber Universitario) lo que Adriana tiene que barrar (poner en falta). No es la destitución de la directora sino su modalidad en el lugar que ocupa lo que le genera malestar. En su contenido hay una demanda de otro trato porque el equipo directivo puede ocupar la función cambiando de semblante y ese cambio no equivaldría a una pérdida de autoridad. Por definición el semblante es la apariencia, y pone de manifiesto:

(...) cierta inconsistencia que estaría en quien está encarnando la función, en tanto es alguien que es llevado a ocupar ese lugar pero que puede hacerlo de diversas maneras. Susana Brignoni lo plantea

\footnotetext{
8 "Agente: es el lugar de quien enuncia y la apariencia, el semblante desde el cual lo hace." (Álvarez en Zelmanovich; 2013 [2010]), Flacso, Clase Nº 6, Ap.: III)
} 
REVISTA DE LA ESCUELA DE CIENCIAS DE LA EDUCACIÓN, AÑO 16, NRO. 15, VOL. 1, ENERO A JUNIO DE 2020. PÁGINAS 73 - 83. ISSN $2362-3349$ (EN LÍNEA). ESCUCHAR PARA SOSTENER LA FUNCIÓN DE EDUCAR APORTES PARA PENSAR LA FUNCIÓN DE EQUIPOS DIRECTIVOS Y PROFESORES DE ORIENTACIÓN Y TUTORÍA SECUNDARIA. IRMA KUNDT.

al diferenciar el encargo social, por ejemplo de ocupar el lugar de profesor o de psicólogo, del modo en que este encargo es asumido (Zelmanovich; 2013 [2010]), Flacso, Clase № 6, Ap.: III).

\section{Esto no me corresponde hacer, esto tampoco. Yo no estudié para esto, esto sí}

Otra arista para pensar el malestar escogido es indagar en cómo se atienden o no los encargos de época en el contexto de la obligatoriedad de la educación secundaria.

Jésica Molina (2018) presenta los hallazgos del equipo de investigación que dirige Perla Zelmanovich en relación a la distinción de los conceptos rol, encargo y función. A partir de sus aportes considero que en las escuelas secundarias de Chubut es un problema la rigidez con la que se piensan y asumen los roles y adhiero al pensamiento de que así declina la función (Zelmanovich, Molina, 2018).

El Rol deriva de lo que está escrito, en el estatuto o contrato de trabajo, es designado por el aparato burocrático, es la especificación de las tareas que cada profesional está llamado a cumplir (Zelmanovich, Molina, 2018). En este sentido, y porque el cargo de POT se encuentra sin nomenclar a diferencia de otros (Preceptor/a, Bibliotecario/a), esta condición hace que exista una cierta indefinición del rol $^{9}$. No obstante, si bien estas especificaciones no están de modo general en un Estatuto, Decreto o Resolución, en su ausencia algunas escuelas avanzan en la escritura, tal el caso de las $\mathrm{PP}^{10}$.

Y en esta falta está su potencia y resulta ventajosa para adoptar una perspectiva situacional (Terigi, 2014) en la que prevalezca la función compartida de pensar en equipo condiciones de enseñanza y atención a la singularidad de cada sujeto y en función de los muchos y complejos problemas a resolver.

Los gremios docentes a veces encarnan una tradición normalista y las invocan incluso siendo incongruentes con nuevos derechos (de estudiantes), pregonan no hacer más o nada que no esté escrito, contribuyen a rigidizar los comportamientos y las posibilidades de pensar. En este caso si bien no hay lo prescripto razonan a partir del tipo de designaciones (horas cátedras o cargo). Como las horas cátedras son todas frente a alumnos/as algunas personas parecen creer que los cargos estarían exceptuados, que para estar en el aula deberían cobrar horas cátedras ¿y por lo tanto no deberían ocuparse de los/as estudiantes en la función de enseñanza, aunque paradójicamente son cargos docentes? Se autorizan y naturalizan que los/as preceptores/as atiendan a los/as alumnos/as sin nada interesante que ofrecerles, que los/as POT acompañen a su PP sin hacerle aportes (explicar, corregir, diseñar una clase, ósea, participar de todo el proceso de enseñanza desde la ideación, a la ejecución y la evaluación compartida). Si un profesor/a falta asumen, entre lo que les toca hacer, "darse una vuelta por el aula", "mirar", "vigilar que nada pase"11 y efectivamente hacen que no pase nada en el sentido de un acontecimiento educativo, no pasa nada porque están desimplicados de la función docente. Desatienden a los chicos y las chicas si no hay contenido a enseñar. Enseñar como parte de un proceso de filiación necesario para su ingreso en la cultura y como una obligación de los mayores en relación a ellos/as. Sin implicación no hay enseñanza y sin enseñanza no hay un cuidado integral y responsable.

En el caso de los/as POT ¿adhieren al mismo razonamiento que realizan preceptores/as y sostienen los gremios?, ¿Por ello al no tener horas cátedras sienten y expresan que no les corresponde integrar equipos para estar en las aulas y dar y sostener la clase?

Con mayor o menor fidelidad a los encargos formulados desde las políticas públicas y coordenadas de época, cada institución especifica algunas tareas (rol) y define la función y los efectos que se espera de quienes lo ocupan.

Por encargo se entienden los pedidos a cada profesional, en relación con los significantes amos de época, ejemplo: "mandato de inclusión". El encargo presenta los ideales que se ponen en juego en cada institución (Zelmanovich; Molina, 2018).

Me resulta oportuno pensar en la potencia-impotencia de estos ideales, por cierto muy diferentes entre escuelas, y a veces algunos apartados del espíritu de la Ley; tanto es así que incluso en algunas constituyen la base de prácticas de exclusión con las que cimientan su "prestigio": no matricular a estudiantes repitentes, derivar a otras escuelas a quienes presentan sobre edad, tomar exámenes de ingreso, etc.

"La función, nos dice Molina, es en cambio lo que se espera a partir del ejercicio del rol, se verifica por sus efectos" (Molina, 2018, s/p). Como efectos de la función en las escuelas que comparten el encargo de la inclusión podría ejemplificar la presencia y permanencia de los/as chicos/as en la escuela, que la habiten y transiten ya sea en su formato habitual o en los trayectos alternativos, y se egresen con título.

Si bien con la obligatoriedad de la educación secundaria aparecen nuevas responsabilidades para los/as adultos/as, aún no aparecen formuladas en estatutos de trabajo y otras normas desactualizadas en

\footnotetext{
${ }^{9}$ En 2019 el Ministerio de Educación de la Provincia del Chubut presenta el Documento Roles y funciones, para la consulta a los/as docentes en las escuelas y supervisión, atento a la necesidad de nomenclar el cargo como requerimiento para establecer los alcances de título. Propone actualizar los roles que acompañan las trayectorias escolares de los estudiantes del nivel.

${ }_{10}^{10}$ El gremio Atech se opone a estas funciones considerándolas formas de flexibilización laboral.

${ }^{11}$ Estas expresiones las recupero de una preceptora; recordaba que en una escuela en la que había trabajado si los estudiantes tenían horas libres la consigna de la directora era "vos andá y miralos, fijate qué están haciendo, que no hagan lío". La expresión entre comillas corresponde a mis crónicas de fraseo.
} 
REVISTA DE LA ESCUELA DE CIENCIAS DE LA EDUCACIÓN, AÑO 16, NRO. 15, VOL. 1, ENERO A JUNIO DE 2020. PÁGINAS 73 - 83. ISSN 2362-3349 (EN LÍNEA). ESCUCHAR PARA SOSTENER LA FUNCIÓN DE EDUCAR APORTES PARA PENSAR LA FUNCIÓN DE EQUIPOS DIRECTIVOS Y PROFESORES DE ORIENTACIÓN Y TUTORÍA SECUNDARIA. IRMA KUNDT.

relación a este marco regulatorio de mayor jerarquía. Por lo expuesto a modo de hipótesis sugiero que el encargo de la inclusión es resistido y está encubierto. La resistencia está latente y opera en los muchos modos de eludir lo que a las personas adultas nos toca hacer: incluir y multiplicar experiencias subjetivantes, pluralizar modos de acoger las diferencias; cumplir la función de educar.

Poco se puede hacer desde comportamientos rígidos que se derivan de prescripciones que clasifican lo que corresponde o no hacer según el rol ("esto sí me corresponde", "esto no me corresponde") y las ideas homogeneizadoras pensadas para todos/as sin variantes.

Se me presenta válido un encuadre y las funciones directivas si son congruentes con el encargo de la inclusión. Un encuadre puede acoger la singularidad de intereses y deseos de los/as profesionales siempre que habilite encuentros en los que florezca la plasticidad para pensar y se planifique con libertad de enseñanza.

\section{Si hay victimización no puede estar implicada la responsabilidad}

(Azubel; 2014, Clase 11, Ap: Presentación)

Desarmonizar incomoda pero es necesario, sobre todo si se trata de educar: "cierta eficacia en la acción transformadora, depende de no tragarse los significantes impuestos por la actualidad, lo que implica estar en un lugar incómodo. Desacomodado". (Azubel; 2014, Clase 11, Ap. III)

Como integrante del equipo directivo sostuve la exigencia de una posición profesional ¿Cómo seguimos si esta idea no va más? Si no hay PP, ¿qué hacemos? No presté atención a la catarsis de Adriana y las POT. Como plantea Azubel la catarsis o simplemente hablar, es diferente de "tomar la palabra". Si hubiesen tomado la palabra habríamos recepcionado ideas en lugar de quejas. Ideas, porque:

(...) no podemos renunciar a la aspiración de aportar para fortalecer las instituciones educativas para que sean aptas para proveer a sus integrantes de una contención y sostén responsable. En esa aptitud para la contención está la responsabilidad de la institución para poder desempeñar sus objetivos sociales, lo que constituye la razón social de la existencia de cada institución (Azubel; 2014, Clase 11, Ap. I).

Creo que hacer depositario al equipo directivo de esta actitud de no escuchar deja afuera a Adriana de toda implicación responsable, y efectivamente en concordancia con este quedar afuera efectivamente renuncia.

Dicha ajenidad o no implicación estaría sostenida por un sentimiento o convicción de impotencia. Es desde ese rol- fortaleza que se autoriza (de un modo no consciente quizás) a escabullir toda respuesta que haga posible virar de una posición de objeto (en la que se está alienado) a una posición activa y de implicación. Cuanto más vaciado de potencia está el propio sujeto, más se alimenta el supuesto poder del Otro (social, político, familiar, simbólico) a quien se dirigen las demandas sin que en las mismas se vean puntos de implicación subjetivo o incluso colectivo (Azubel; 2014, Clase 11, Ap. I).

\section{La Ley de Educación Nacional 26.206 y la responsabilidad pública}

En este contexto la educación trabaja en territorios que Violeta Núñez (2014) describe en términos "de frontera", para referir a sus contornos borrosos, entre inclusión-exclusión social, debido a la amenaza permanente $y / 0$ acto de pasaje de la primera a la segunda categoría.

La Ley atiende a la emergencia de una nueva responsabilidad pública hacia las infancias y adolescencias, para que la asistencia de niños/as y adolescentes pueda sostenerse, realizarse y mantenerse en el tiempo con mejores posibilidades: "hay una responsabilidad social en juego ante la transmisión cultural necesaria para los niños y adolescentes en general y, muy particularmente, para aquéllos que se encuentran en situación de fragilidad o vulnerabilidad social" (Núñez; 2014, Clase 10, Ap. III).

La escuela ha sido y es objeto de un conjunto de demandas, entre ellas hoy recibe el encargo de la inclusión, pero, en los hechos encontramos una activa resistencia latente que se juega a diario en muchos modos de desatención. Creo que Adriana, inconscientemente, y algunos/as POT encarnan esa posición; por ello crear o participar en la ideación de diferentes dispositivos de ayuda no es algo que realicen de modo espontáneo y consideran paternalista "dar más oportunidades a quienes no la merecen porque especulan con la nota" 12 .

\footnotetext{
${ }^{12}$ La expresión entre comillas corresponde a mis crónicas de fraseo.
} 
REVISTA DE LA ESCUELA DE CIENCIAS DE LA EDUCACIÓN, AÑO 16, NRO. 15, VOL. 1, ENERO A JUNIO DE 2020. PÁGINAS 73 - 83. ISSN $2362-3349$ (EN LÍNEA). ESCUCHAR PARA SOSTENER LA FUNCIÓN DE EDUCAR APORTES PARA PENSAR LA FUNCIÓN DE EQUIPOS DIRECTIVOS Y PROFESORES DE ORIENTACIÓN Y TUTORÍA SECUNDARIA. IRMA KUNDT.

Su pérdida de interés, no querer saber acerca de algo (x contenido, de x disciplina), no saber qué hacer o cómo dar continuidad a una clase en caso de no contar con su PP, manifestar ausencia de disponibilidad para aprender en equipo de PP, hacer equipo sin entusiasmo, me remite a las ideas de Zelmanovich, y aunque ella piensa en relación con los/as estudiantes, encuentro algo de ello entre algunas personas adultas y considero atinado transferir su hipótesis al caso que me ocupa.

La pérdida de interés estaría anclada en las referencias que ofrece la cultura escolar tradicional, cultura en la que no hay equipos de trabajo en las aulas (lo contrario a integrar PP) y en la cual prevalece una organización fragmentada y estructurada del espacio y el tiempo para la enseñanza de disciplinas estancas a cargo de un profesor que cobra para eso (lo contrario a las experiencias de aula acelerada y ClIC). Enseñar sería trabajo de otros/as, no el de preceptores/as, no el de POT. En este sentido:

No se trata de un "menos" (a-patía), ni de aquello que les falta (el interés), o de un elemento que se ha perdido (el entusiasmo). Se trata de un "más", de un tapón (...) un indicio de "alienación" respecto de los referentes conocidos (...) dan muestras de "ponerse en guardia" ante el riesgo de perder esa referencia, aunque eso los mantenga en una posición pasiva. (Zelmanovich; 2014, Clase 4, Ap. V)

\section{De la fijeza en la posición a la rotación del discurso}

El equipo directivo persistía con el encuadre. Consideré que cuando cada adulto/a abdica destituyéndose nadie se ocupa del encargo de la inclusión y los/as chicos/as quedan en banda (Duschatzky y Corea; 2014).

Al final de este recorrido pienso en la fijeza de mi posición (y la de mi equipo directivo) y en el sentido de la repetición de la racionalización como el mecanismo de defensa empleado una y otra vez para "no dar lugar a dudas", dudas que posiblemente remiten a mi propia inseguridad en el ejercicio del rol y que necesitó la afirmación "la directora soy yo" y "este es el encuadre".

No obstante ya no sirve endilgarme toda la culpa. Tampoco depositarla en Adriana e ir de un lado al otro con movimientos pendulares. Sí pensar un desencuentro, y pensar en las posiciones, puesto que, parafraseando a Voltolini (2014), en la danza de los discursos no baila una sola persona. Asimismo recuperar el concepto de reunión que aporta Zelmanovich; nos explica cómo se tejen las operaciones en la relación entre el sujeto y el Otro.

El ser del sujeto, que recibe sus sentidos del Otro, se produce en un tipo de operación lógica que es de reunión. El producto de la reunión no es ni lo uno ni lo otro, es producto de lo que falta de un lado y lo que falta del otro. El sujeto y su verdad es efecto de esa superposición de faltas, que emerge como sinsentido (...) El sujeto encuentra una falta en el Otro, en la intimación que ejerce sobre él el Otro con su discurso (Zelmanovich; 2014, Clase 4, Ap: I).

Por otra parte, a partir de este concepto de reunión, pienso en la rotación en relación con POT y con Adriana. Persisto pensando en ella porque sigo en el equipo y creo que nuevos hallazgos pueden ayudarnos a escuchar y virar en el discurso cuando declina la función. Escuchar como un gesto de hospitalidad.

La llegada a un sitio nuevo, así como el recibimiento, es a producir. No va de suyo, no es algo dado porque estén pensadas las condiciones de recibimiento, que el alojamiento ocurra. Aunque es necesario el marco de lo establecido para dar una organización a aquello que ocurrirá, no podemos pensar que lo que ocurrirá se adaptará punto por punto a ese marco. Lo que ocurre en cada comienzo (...) ocurre como nuevo y lo nuevo introduce cambios. Recibir lo nuevo cambia (Balbi y Serravalle; 2014, Clase 2, Ap. II).

Adriana no hace lazo con el Otro que encarna la Ley (discurso del amo) y que la presenta y sostiene desde un discurso universitario sin cambiar de semblante, discursos que le resultan insoportables porque los efectos o su producción es ponerla en el lugar del no saber, y en su eficacia es el lugar que efectivamente encarna una y otra vez: "Es a través del discurso que el sujeto tiene una chance de encontrar al otro, al mismo tiempo que lo pierde en alguna medida, allí donde construye su diferencia" (Zelmanovich; 2013 [2010]), Flacso, Clase $\mathrm{N}^{\circ}$ 6, Ap.: II).

Pienso el sostenimiento. El semblante del equipo directivo pudo y puede ser distinto, cobrar otras formas, unas nuevas que produzcan recibimiento y alojen las singularidades de cada profesional que se suma al equipo. Invento una metáfora, imagino formas más abrazadoras, y al describirlas con este calificativo pienso en el sentido del término emparentado con el abrazo como algo que pone a las personas en una situación de mayor proximidad, lo que es distinto a la distancia que pone una posición de todo saber. Siguiendo con la metáfora el abrazo no es tensionante, si lo comparamos con tensores que también pueden sostener, pero lo hacen desde afuera, desde unos extremos sin mayor contacto. 
REVISTA DE LA ESCUELA DE CIENCIAS DE LA EDUCACIÓN, AÑO 16, NRO. 15, VOL. 1, ENERO A JUNIO DE 2020. PÁGINAS 73 - 83. ISSN 2362-3349 (EN LÍNEA). ESCUCHAR PARA SOSTENER LA FUNCIÓN DE EDUCAR APORTES PARA PENSAR LA FUNCIÓN DE EQUIPOS DIRECTIVOS Y PROFESORES DE ORIENTACIÓN Y TUTORÍA SECUNDARIA. IRMA KUNDT.

\section{Del escritorio de la dirección a la mesa del Rey Arturo}

La proximidad abrazadora que pienso no es otra sino aquella que pone a un equipo en mesas de trabajo una y otra vez, mesas circulares como las del Rey Arturo, en las que, como Arturo, se sientan los/as directivos/as de una institución en lugar de delegar y notificar por escrito cuál es el encuadre. Sentarse a pensar con el otro/a antes que delegar tareas previamente pensadas sin ellos/as.

En la mesa redonda el radio que separa a cada quien del centro es el mismo: todos saben, todos escuchan, proponen, hablan, deciden. Eso es vital para luego poner en marcha un proyecto. Será necesario sobre la marcha volver a sentarse y conversar y evaluar y otra vez decidir.

En este dispositivo el énfasis de la metáfora está en la mesa redonda y no en la persona del Rey.

También la pareja pedagógica puede resultar un encuadre poderoso si se propician una y otra vez encuentros para la planificación de la tarea. Este tiempo en Provincia de Chubut no está previsto para profesores/as, y a consecuencia de ello las personas con cargos (POT, PEP) afectados a emplear parte de su tiempo en este dispositivo se ven desvalidas si no han tenido el tiempo de sentarse con la persona que acompañarán en una disciplina específica y frente a estudiantes.

Los directivos deben entonces organizar estas agendas garantizando los encuentros con la frecuencia que las PP necesitan. Pienso asimismo que esas reuniones requieren por una parte la intimidad de la PP y la libertad para organizar la enseñanza. Y también deben preverse tiempos para compartir con directivos los resultandos alcanzados en sus planificaciones ya que tampoco pueden estar desimplicados de las propuestas educativas que brinda la institución de la que son responsables.

Por muy democrático que resulte hacerlo así no debemos olvidar que todo encuentro conlleva un desencuentro por estructura y la mesa redonda no será tarea sencilla pero el ejercicio de alojamiento del otro con la diferencia que porta, eso mismo, por complejo que sea, no nos robotiza. Nos humaniza. Con proximidad se gana en humanidad lo que seguramente se pierde en las alturas del panóptico.

\section{Conclusiones provisorias}

¿Para sostener y alojar en la tarea a Adriana y su deseo se trataba de abandonar el encuadre?, ¿escuchar a Adriana equivalía a desistir de la PP?

Bosquejar el dispositivo-metáfora propuesto me hizo pensar que tal vez la demanda de Adriana pudo atenderse con ello.

Nótese que en la metáfora hay rey, o sea no equivale a la destitución de la dirección de una escuela ni a la falta de encuadre, pero sí a sus modalidades de sostén.

Escuchar es un gesto de hospitalidad que no se logra sin sortear las distancias, hacer espacios, encontrar tiempos. El equipo directivo debe gestionarlos y garantizarlos para que las reuniones sostenidas posibiliten los encuentros y analizar los problemas, intercambiar ideas, soluciones, idear proyectos, evaluarlos, pensarlos. Para que cada quien experimente el poder de hacerlo con otros/as y la potencia que deviene de la experiencia colectiva, contra tanto individualismo aprendido.

\section{Referencias bibliográficas}

Azubel, A. (2014). "Acerca del lugar de la víctima: Responsabilidad $\diamond$ victimización.". Clase 11. Seminario III. Especialización "Psicoanálisis y prácticas socioeducativas". FLACSO Argentina. Disponible en: flacso.org.ar/flacso-virtual

Balbi, C. y Serravalle, L. (2014). "La estructura de la palabra en psicoanálisis.” Clase 2, Seminario I. Especialización "Psicoanálisis y prácticas socioeducativas", FLACSO Argentina, disponible en: flacso.org.ar/flacso-virtual

Kait, G. (2014). "Tres registros para leer la estructuración del sujeto: imaginario, simbólico y real”, Clase 1, Seminario I. Especialización "Psicoanálisis y prácticas socioeducativas", FLACSO Argentina, disponible en: flacso.org.ar/flacso-virtual

Kundt, I. (2018). Escuela de verano en el contexto de la educación secundaria obligatoria. Narrativa de una experiencia pedagógica. El profesional en Ciencias de la Educación en la función de Supervisor de escuela. Revista de la Escuela de Ciencias de la Educación - Vol. 2, núm. 13. Páginas 89-105.Recuperado de: https://rephip.unr.edu.ar/xmlui/handle/2133/14576

Molina, Y. y Zelmanovich, P. (2017). "Rol, encargo y función: aportes para deconstruir la posición", Clase 9 , Seminario II. Especialización "Psicoanálisis y prácticas socioeducativas", FLACSO Argentina, disponible en: flacso.org.ar/flacso-virtual, recuperado en 2018.

Nuñez, V. (2010). "La Pedagogía Social. Una apuesta educativa que propone modalidades de vínculo social, nuevas maneras de repartir el juego social y de atender las responsabilidades públicas en épocas de retracción del Estado". Diplomatura Superior en Psicoanálisis y Prácticas Socioeducativas. FLACSO. Buenos Aires.

Terigi, F. (2014) "Discurso normativo y prácticas normalizadoras en el sistema educativo." Clase 6, Seminario II. Especialización "Psicoanálisis y prácticas socioeducativas", FLACSO Argentina, disponible en: flacso.org.ar/flacso-virtual

Voltolini, R. (2014) "Educación inclusiva y régimen de excepción." Clase 5, Seminario II. Especialización "Psicoanálisis y prácticas socioeducativas", FLACSO Argentina, disponible en: flacso.org.ar/flacso-virtual 
REVISTA DE LA ESCUELA DE CIENCIAS DE LA EDUCACIÓN, AÑO 16, NRO. 15, VOL. 1, ENERO A JUNIO DE 2020. PÁGINAS 73 - 83. ISSN 2362-3349 (EN LíNEA). ESCUCHAR PARA SOSTENER LA FUNCIÓN DE EDUCAR APORTES PARA PENSAR LA FUNCIÓN DE EQUIPOS DIRECTIVOS Y PROFESORES DE ORIENTACIÓN Y TUTORÍA SECUNDARIA. IRMA KUNDT.

Zelmanovich, P (2014) "La constitución del sujeto en un tiempo lógico y discontinuo, en los desfiladeros de dos operaciones: alienación separación.". Clase 4, Seminario I. Especialización "Psicoanálisis y prácticas socioeducativas", FLACSO Argentina, disponible en: flacso.org.ar/flacso-virtual

(2013 [2010]). "Leer el lazo con la teoría de los cuatro discursos. Una herramienta de trabajo", Clase $N^{\circ} 6$ (virtual). Módulo 2: Avatares del lazo social en los fundamentos de la producción subjetiva. Lo familiar, lo fraterno, lo educativo. Diploma Superior en Psicoanálisis y Prácticas Socio - Educativas, Cohorte 7, FLACSO Argentina disponible en: flacso.org.ar/flacso-virtual, recuperado en 2018. 